\title{
BPSK to ASK Signal Conversion Using Injection-Locked Oscillators-Part I: Theory
}

\author{
José María López-Villegas, Senior Member, IEEE, and Javier Jose Sieiro Cordoba, Member, IEEE
}

\begin{abstract}
This paper presents a new method and circuit for the conversion of binary phase-shift keying (BPSK) signals into amplitude shift keying signals. The basic principles of the conversion method are the superharmonic injection and locking of oscillator circuits, and interference phenomena. The first one is used to synchronize the oscillators, while the second is used to generate an amplitude interference pattern that reproduces the original phase modulation. When combined with an envelope detector, the proposed converter circuit allows the coherent demodulation of BPSK signals without need of any explicit carrier recovery system. The time response of the converter circuit to phase changes of the input signal, as well as the conversion limits, are discussed in detail.
\end{abstract}

Index Terms-Amplitude shift keying (ASK), bifurcation, converters, injection-locked oscillator (ILO), phase shift keyings (PSKs).

\section{INTRODUCTION}

$\mathbf{T}$ HE DIGITAL phase shift keying (PSK) of a sinusoidal signal is one of the most efficient modulation techniques, both in terms of noise immunity and required bandwidth. Coherent demodulation is the preferred procedure to demodulate PSK signals, especially when optimum error performance is of particular importance [1]. Coherent demodulation requires the availability of a local carrier having the same frequency and phase than the received modulated carrier. However, frequency and/or phase deviations degrade the detection process and, consequently, the system performance. Therefore, local carrier synchronization is a critical issue in most digital communication systems. Carrier recovery is accomplished by using synchronization loops [2]-[5]. The most widely used are the squaring loop and Costas loop, shown in Fig. 1(a) and (b), respectively. However, synchronization time is usually large, leading to loss of data at the beginning of a communication or malfunctioning in burst mode transmissions. Noncoherent demodulation of PSK signals can overcome this problem, however, noise immunity is worst and the bit period has to be known [1].

This study proposes an alternative method for the demodulation of binary phase-shift keying (BPSK) signals, which is based on the use of a coherent BPSK to amplitude shift keying (ASK) converter [6], as depicted in Fig. 2. A simple envelope detector cascaded to the converter acts as the final stage of the demodulation system. The operation of the BPSK to ASK converter

Manuscript received January 31, 2005; revised July 8, 2005. This work was supported by the Spanish Science and Technology Commission under Project TIC2001-2947-C02-01.

The authors are with the RF Group, Department of Electronics, University of Barcelona, E-08028 Barcelona, Spain (e-mail: josem@el.ub.es).

Digital Object Identifier 10.1109/TMTT.2005.859875
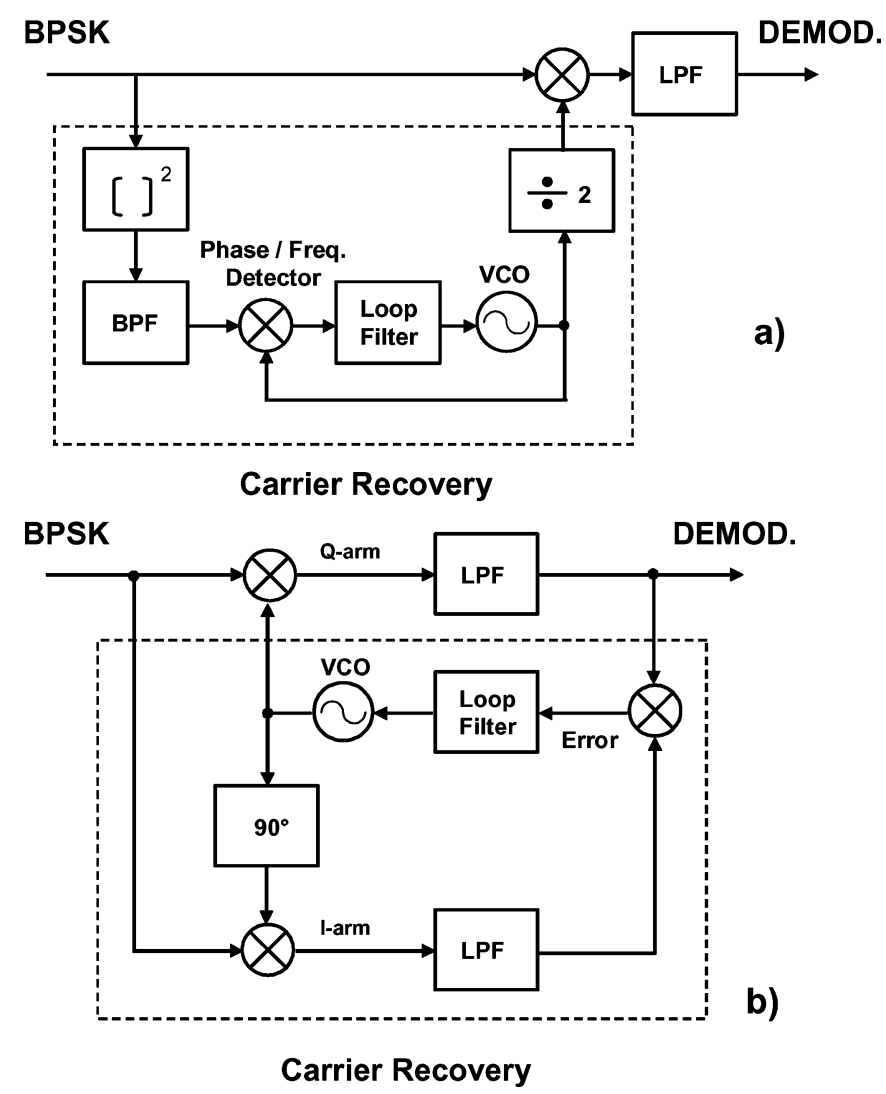

Fig. 1. Block diagram of typical BPSK demodulation schemes using carrier recovery systems. (a) Squaring loop. (b) Costas loop.

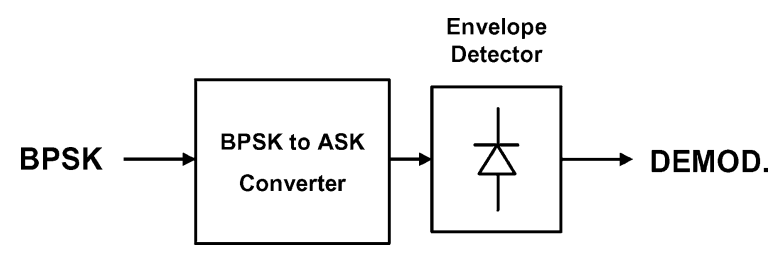

Fig. 2. Block diagram of the proposed BPSK demodulation scheme.

relies on two main principles: the superharmonic injection of oscillators and interference phenomena. The former is used to lock the oscillators (in frequency and phase) with the incoming signal, whereas the later is used to generate an amplitude interference pattern that reproduces the original phase modulation. Section II is devoted to the analysis of injection-locked oscillators (ILOs), whereas Section III covers the conversion mechanism, dynamics, and limitations of the coherent BPSK to ASK converter. 


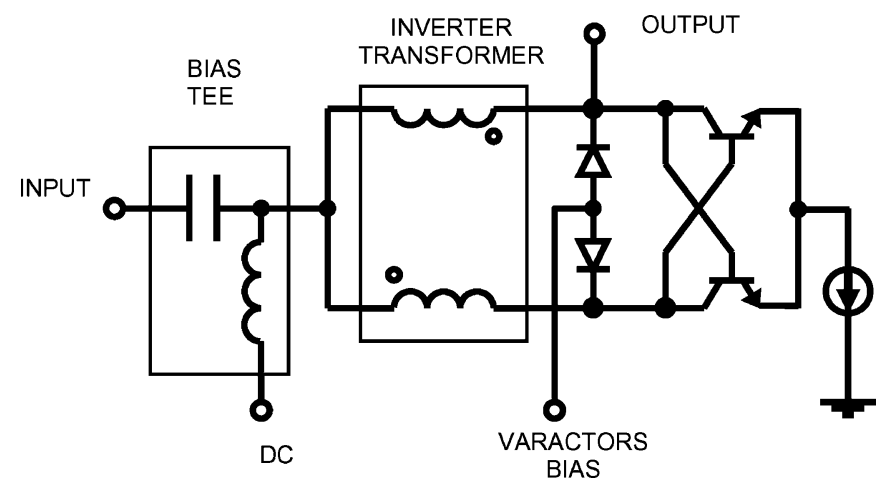

Fig. 3. Circuit schematic of a second harmonic ILO.

\section{ILOs}

Injection is a usual way to synchronize an oscillator with an incident signal. The injection-locking mechanism, first analyzed by Van der Pol in 1927 [7], has been investigated by several authors [8]-[11]. When an injected signal is close to the $N$ harmonic of the free running frequency of the oscillator, the ensemble is known as superharmonic or $N$ th harmonic ILO. In the locked state, superharmonic ILOs act as frequency dividers, with the dividing factor being the nearest harmonic order of the ILO to the injected signal. For that reason, these components are also known as injection-locked frequency dividers (ILFDs) [12], [13]. The output of a superharmonic ILO or ILFD could be in any of $N$ possible phase states ( $N$ being the harmonic order). This is due to the phase uncertainty introduced by the process of frequency division. For example, in the case of a second harmonic ILO, the output frequency is half the frequency of the injected signal and phase uncertainty is equal to $\pi$.

In this study, the interest is focused in a particular implementation of a second harmonic ILO, of which a schematic is shown in Fig. 3. The circuit is a cross-pair oscillator, whose resonant tank consists of an inverter transformer and a pair of varactor diodes. The input port of the injected signal is the center tap of the transformer. Ideally, under common-mode excitation, the transformer acts as a short circuit, therefore, the injected signal is found without distortion at the terminals of the varactors. Due to the nonlinear behavior of the stored charge dependence up on the applied voltage, the injected signal, at frequency $2 \cdot f_{\text {lock }}$, mixes with the oscillator signal at frequency $f_{r}$ close to $f_{\text {lock }}$. As a consequence, a new current component appears, which modifies the characteristics of the resonant tank. In order to quantify this process, let us consider a second-order approximation for the stored charge versus applied voltage at the varactors

$$
Q=Q_{o}+C_{o} V+\frac{\alpha}{2} V^{2}+\ldots
$$

where $Q_{o}$ is a constant charge depending on the bias conditions, $C_{o}$ is the small-signal capacitance at the bias point, $V$ is the applied voltage, and $\alpha$ is a second-order coefficient evaluated at the bias point of the varactors.

According to (1), the current $I$ passing through the varactors is given by

$$
I=\frac{d Q}{d t}=C_{o} \frac{d V}{d t}+\alpha V \frac{d V}{d t} .
$$

In a first approximation, the applied voltage $V$ is the addition of the injected signal $V_{2}$ and the fundamental component of the oscillation signal $V_{1}$, which can be expressed as follows:

$$
\begin{aligned}
& V_{1}=A_{1} \cos \left(2 \pi f_{\text {lock }} t+\Phi(t)\right) \\
& V_{2}=A_{2} \cos \left(4 \pi f_{\text {lock }} t+\psi\right)
\end{aligned}
$$

where $A_{1}$ is the voltage amplitude of the fundamental component of the oscillator, $A_{2}$ is the voltage amplitude of the injected signal, $f_{\text {lock }}$ is the frequency in the locked state, and $\Phi(t)$ and $\psi$ are the corresponding phases. Note that the time dependence of $\Phi(t)$ takes into account the evolution of the oscillator frequency from the free-running state to the locked state (i.e., at $t=0,2 \pi f_{\text {lock }}+d \Phi / d t=2 \pi f_{r}$, with $f_{r}$ being the oscillator's free-running frequency).

Provided that $|d \Phi / d t| \ll 2 \pi f_{\text {lock }}$ at any time $t$, i.e., $f_{\text {lock }} \approx$ $f_{r}$, by substituting $V=V_{1}+V_{2}$ in (2) and after some calculation to evaluate the mixing terms, one can obtain the following expression for the current at the fundamental frequency $I_{1}$ passing through the varactors:

$$
I_{1}=\left[C_{o}+\alpha \frac{A_{2}}{2} \sin (\theta(t))\right] \frac{d V_{1}}{d t}+-\alpha A_{2} \pi f_{\mathrm{lock}} \cos (\theta(t)) V_{1}
$$

where angle $\theta(t)$ is given by

$$
\theta(t)=2 \Phi(t)-\psi+\frac{\pi}{2} .
$$

The first term in (4) is the displacement current related to the capacitance of the varactors. The second term is an in-phase current, which can be positive (i.e., dissipative) or negative (i.e., regenerative) depending on the value of angle $\theta(t)$. This current is responsible for the oscillation rise in parametric analog frequency dividers [12].

According to (4), the capacitance of the varactors in the locked state $C(\theta)$ is given by

$$
C(\theta)=C_{o}+\alpha \frac{A_{2}}{2} \sin (\theta(t)) .
$$

$C(\theta)$ changes depending on the amplitude of the injected signal $A_{2}$ and angle $\theta(t)$. Therefore, it implies a change in the oscillator frequency, which is reflected in $\phi(t)$ and, through (5), also back in $\theta(t)$. The differential equation governing the dynamics of this process can be obtained through the instantaneous oscillation frequency

$$
2 \pi f_{\text {lock }}+\frac{d \Phi(t)}{d t}=\frac{1}{\sqrt{L C(\theta)}}
$$

where $L$ is half the inductance of the inverter transformer working in differential mode. Taking into account that $2 \pi f_{r}=1 / \sqrt{L C(\theta)}, d \theta=2 d \Phi$, and $\left|\alpha A_{2}\right| \ll C_{o}$ (i.e., $f_{\text {lock }} \approx f_{r}$ ), one finally obtains

$$
\frac{d \theta(t)}{d t}=4 \pi\left(f_{r}-f_{\mathrm{lock}}\right)-\pi f_{r} \frac{\alpha A_{2}}{C_{o}} \sin (\theta(t)) .
$$

Equation (8) explains the dynamic of the locking process through the evolution of $\theta(t)$ over time. It establishes the steady- 


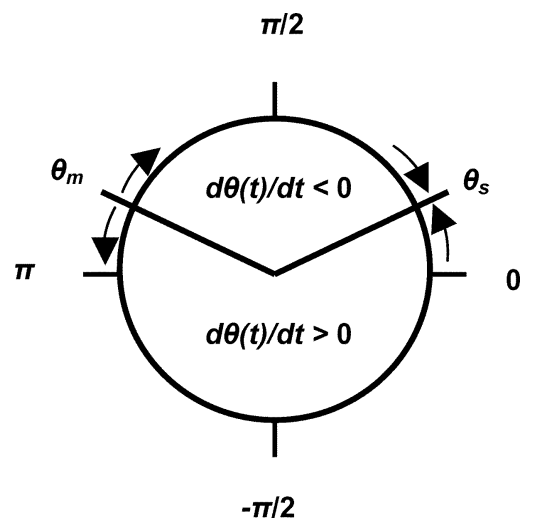

Fig. 4. Graphical representation of the stability analysis of (8) in the steady state.

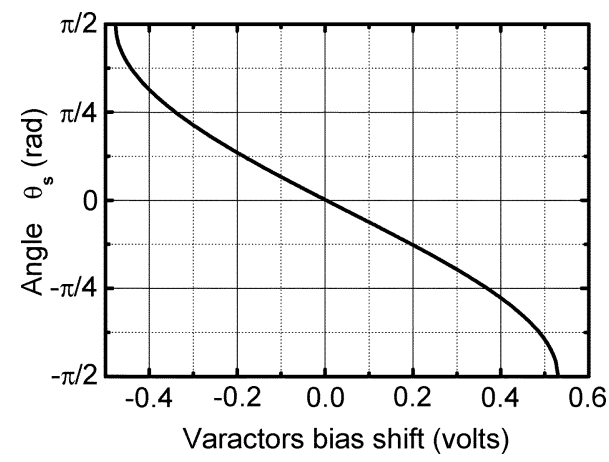

Fig. 5. Steady-state solution of (8) versus varactors bias shift.

state condition, locking range, locking sensitivity, and dynamic behavior.

\section{A. Steady-State Solution}

In the steady state, the value of $d \theta(t) / d t$ is 0 . Thus, the solutions of (8) are two fixed values of angle $\theta$

$$
\theta_{s}=\arcsin \left(\frac{4 C_{o}}{\alpha A_{2}} \frac{f_{r}-f_{\text {lock }}}{f_{r}}\right)
$$

and

$$
\theta_{m}=\pi-\theta_{s}
$$

As is schematically depicted in Fig. 4, any perturbation around $\theta_{s}$ will be automatically compensated. Consequently, $\theta_{s}$ is stable. On the contrary, any perturbation around $\theta_{m}$ will be amplified, indicating that it is an unstable value.

According to (9), the second harmonic ILO shown in Fig. 3 can be used as a continuous phase modulator [14]. In the locked state, the output frequency of the second harmonic ILO is always half the injected frequency. A change in the varactors bias conditions then produces a phase shift instead of a frequency shift. This fact can be observed in Fig. 5, where angle $\theta_{s}$ has been plotted against the varactor's bias shift. To evaluate $C_{o}, \alpha$, and $f_{r}$, the depletion capacitance formula of a $\mathrm{p}-\mathrm{n}$ junction has been used. Moreover, the zero of the bias shift scale corresponds to the bias value at which $f_{r}=f_{\text {lock }}$ (i.e., $\theta_{s}=0$ ).
A quasi-linear behavior is observed for small values of the varactors bias shift. Note that the maximum and minimum achievable values of $\theta_{s}$ are $\pi / 2$ and $-\pi / 2$, respectively, which, according to (5), leads maximum and minimum output phase $\Phi(t)$ of $\pi / 4$ and $-\pi / 4$.

\section{B. Locking Range and Locking Sensitivity}

The locking range of the second harmonic ILO can easily be obtained from (9). The argument of the arcsin function must be kept from -1 to 1 , thus,

$$
\Delta f_{\max }=\left|f_{r}-f_{\text {lock }}\right|_{\max }=f_{r}\left|\frac{\alpha A_{2}}{4 C_{o}}\right| .
$$

A similar expression has been previously reported by other authors [12]. It should be noted that, in contrast with the fundamental locking analysis [8], [9] the second harmonic injection-locking leads to a locking range not dependent on the resonator quality factor. This is due to the fact that injection at the second harmonic does not force the oscillator to oscillate out of its natural or resonant frequency, but actually it changes this frequency.

Equation (11) also establishes a relationship between the frequency shift $\Delta f$ and the minimum injected power $P_{\text {inj,min }}$ required for locking. According to (11), one can expect

$$
P_{\text {inj, } \min } \alpha\left(\frac{\Delta f_{\max }}{f_{r}}\right)^{2} .
$$

That is to say, the locking sensitivity varies as the relative frequency shift to the square.

\section{Dynamic Behavior}

To analyze the dynamic behavior of the locking process, we need to solve (8). Depending on the boundary conditions, we can consider several cases as follows.

1) Phase Locking: In this case, $f_{\text {lock }}=f_{r}$ (i.e., $\theta_{s}=0$ ) and (8) reduces to

$$
\frac{d \theta(t)}{d t}=-\pi f_{r} \frac{\alpha A_{2}}{C_{o}} \sin (\theta(t)) .
$$

Its solution can be written as

$$
\tan \left(\frac{\theta(t)}{2}\right)=\tan \left(\frac{\theta_{o}}{2}\right) \exp \left(-\frac{t}{\tau}\right)
$$

where $\theta_{0}$ denotes the initial condition of the phase for $t=0$ The time $\tau$ is given by

$$
\tau=\frac{1}{\pi f_{r}} \frac{C_{o}}{\alpha A_{2}} .
$$

Fig. 6 shows the transient responses of $\theta(t)$ for different values of $\theta_{0}$ as a function of the normalized time $t / \tau$. In this particular case, regardless of the injected power, the phase locking always takes place. According to (14), (15), and Fig. 6 , the input power only determines the speed at which equilibrium conditions are reached, i.e., the higher the power, the faster the response. Consequently, provided the injection time is long 


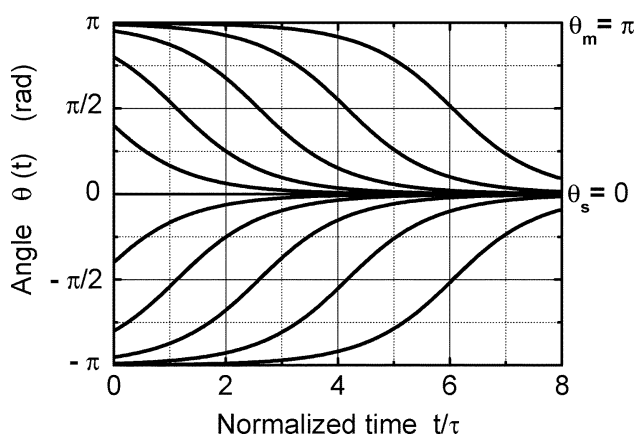

Fig. 6. Time-dependent solution of (8) in the case of phase locking.

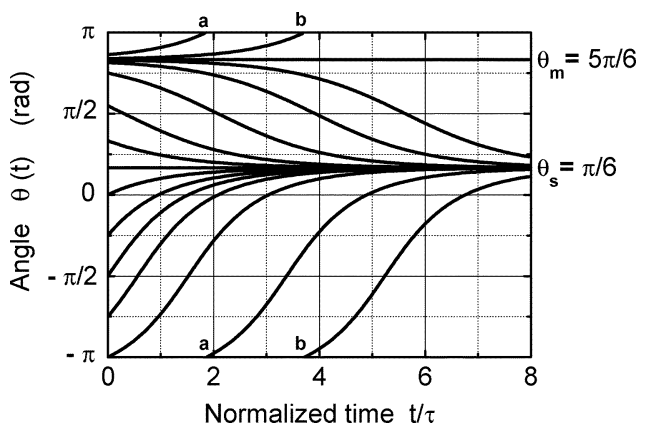

Fig. 7. Time-dependent solution of (8) in the case of phase and frequency locking.

enough, any signal at frequency $2 \cdot f_{r}$ (for instance noise) can lock the oscillator and change its output phase.

2) Phase and Frequency Locking: This case corresponds to the double condition $f_{\text {lock }} \neq f_{r}$ and $4\left|f_{r}-f_{\text {lock }}\right|<$ $f_{r}\left|\alpha A_{2} / C_{0}\right|$. The solution of (8) is now given by

$$
\tan \left(\frac{\theta(t)}{2}\right)=\beta \pm \sqrt{\beta^{2}-1} \frac{1+\gamma \exp \left(-\frac{t}{\tau} \sqrt{1-\frac{1}{\beta^{2}}}\right)}{1-\gamma \exp \left(-\frac{t}{\tau} \sqrt{1-\frac{1}{\beta^{2}}}\right)}
$$

where

$$
\beta=\frac{1}{\sin \left(\theta_{s}\right)}
$$

and

$$
\gamma=\frac{\beta \pm \sqrt{\beta^{2}-1}-\tan \left(\frac{\theta_{o}}{2}\right)}{\beta \mp \sqrt{\beta^{2}-1}-\tan \left(\frac{\theta_{o}}{2}\right)} .
$$

The upper sign applies when $f_{r}<f_{\text {lock }}$ (i.e., $-\pi / 2<\theta_{s}<0$ ), and the lower sign when $f_{r}>f_{\text {lock }}$ (i.e., $0<\theta_{s}<\pi / 2$ ).

Fig. 7 shows an example of the transient response of $\theta(t)$ for different values of $\theta_{0}$ as a function of normalized time $t / \tau$. In this example, angle $\theta_{s}=\pi / 6$. It is clearly observed in Fig. 7 that angle $\theta_{s}$ acts as an attractor of the dynamic phase trajectories while angle $\theta_{m}$ acts as a scatterer. As in previous case 1), the injected power determines the speed at which the equilibrium conditions are reached. However, now a minimum injected

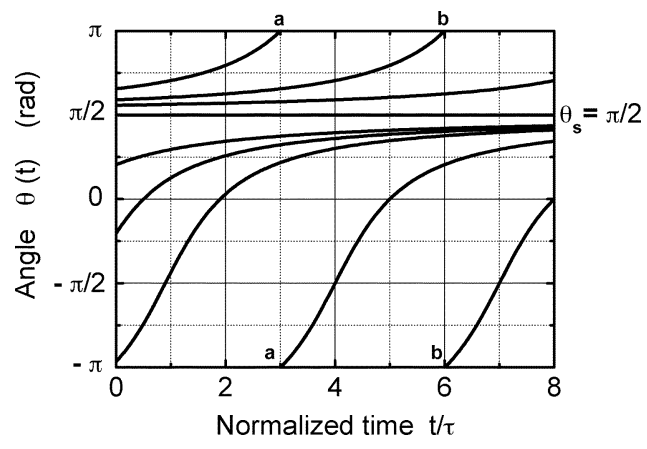

Fig. 8. Time-dependent solution of (8) in the locking threshold.

power is required to assure locking. This situation will be analyzed in the next case.

3) Locking Threshold: This case corresponds to the condition $4\left|f_{r}-f_{\text {lock }}\right|=f_{r}\left|\alpha A_{2} C_{0}\right|$, i.e., $\theta_{s}= \pm \pi / 2$. Here, (8) can be expressed as follows:

$$
\frac{d \theta(t)}{d t}=\frac{\mp 1-\sin (\theta(t))}{\tau} .
$$

The minus sign applies when $f_{r}<f_{\text {lock }}$ and the plus sign applies when $f_{r}>f_{\text {lock }}$. Under these conditions, the solution of (19) is given by

$$
\tan \left(\frac{\theta(t)}{2}\right)=\mp \frac{\gamma-1+\frac{t}{\tau}}{\gamma+1+\frac{t}{\tau}}
$$

where

$$
\gamma=\frac{1 \mp \tan \left(\frac{\theta_{o}}{2}\right)}{1 \pm \tan \left(\frac{\theta_{o}}{2}\right)} .
$$

Once again, in these equations, the upper sign applies when $f_{r}<f_{\text {lock }}$ (i.e., $\theta_{s}=-\pi / 2$ ), and the lower sign applies when $f_{r}>f_{\text {lock }}$ (i.e., $\theta_{s}=-\pi / 2$ ).

The transient response of $\theta(t)$ for different values of $\theta_{0}$ as a function of normalized time $t / \tau$ is shown in Fig. 8. In this case, $\theta_{s}$ is equal to $\pi / 2$. At the locking threshold, the injected power is the minimum required to assure locking. Under these conditions, the ILO is very unstable. Consequently, any perturbation at the input (for instance, noise) can unlock the oscillator.

4) Unlocking: In this case, $4\left|f_{r}-f_{\text {lock }}\right|>f_{r}\left|\alpha A_{2} / C_{0}\right|$. Consequently, $d \theta(t) / d t \neq 0$ at any time $t$, and locking does not occurs, i.e., the injected power is not big enough to assure the locking of the oscillator. Under these conditions, the solution of (8) is given by

$$
\tan \left(\frac{\theta(t)}{2}\right)=\frac{1}{\beta} \mp \sqrt{1-\frac{1}{\beta^{2}}} \quad \tan \left(\frac{t}{2 \tau} \sqrt{\beta^{2}-1}+\gamma\right)
$$

where

$$
\beta=\frac{f_{r}-f_{\text {lock }}}{f_{r}} \frac{4 C_{o}}{\alpha A_{2}}, \quad|\beta|>1
$$




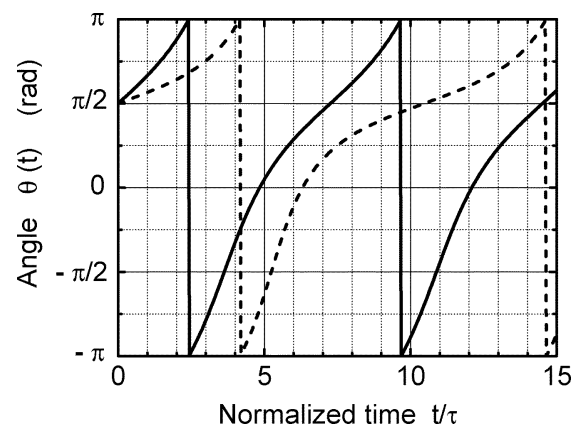

Fig. 9. Time-dependent solution of (8) in the unlocked state. The continuous line corresponds to $\beta=2$ and the dashed line corresponds to $\beta=1.25$.

a)

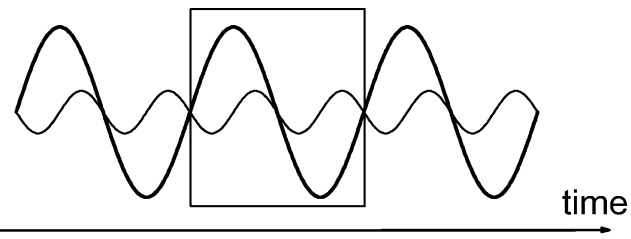

b)

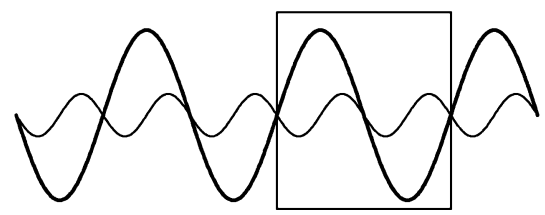

Fig. 10. Schematic representation of voltage waveforms at varactors leads. The thick line corresponds to the fundamental component and the thin line corresponds to the injected signal. (a) and (b) identify the two possible states due to the phase uncertainty. Marker squares have been inserted to highlight that the same phase relationship between the fundamental component and injected signal is observed in both cases.

and

$$
\gamma=\arctan \left[\frac{\beta \tan \left(\frac{\theta_{o}}{2}\right)-1}{\sqrt{\beta^{2}-1}}\right] .
$$

The minus sign applies when $f_{r}<f_{\text {lock }}$ and the plus sign applies when $f_{r}>f_{\text {lock }}$.

Fig. 9 depicts the dynamics of $\theta(t)$ as a function of the normalized time $\tau$ for two values of parameter $\beta$. The continuous line corresponds to $\beta=2$ and the dashed line corresponds to $\beta=1.25$. For comparative purposes, both curves have the same initial conditions $\theta_{0}$. It can be observed that $\theta(t)$ shows a periodic behavior of which the period depends on the $\beta$ parameter. As $|\beta|$ approaches 1 , the period increases and becomes infinity for $|\beta|=1$. This limit situation corresponds to the previous analysis case of the locking threshold.

\section{BPSK TO ASK CONVERSION}

\section{A. Conversion Mechanism}

Let us consider a second harmonic ILO injected by a reference signal. Frequency and power of the injected signal are such as to assure both frequency and phase locking of the oscillator. According to (5), there are two possible values of the output phase $\Phi$ that verify the locking conditions, which differ in $\pi$. This is schematically depicted in Fig. 10. a)

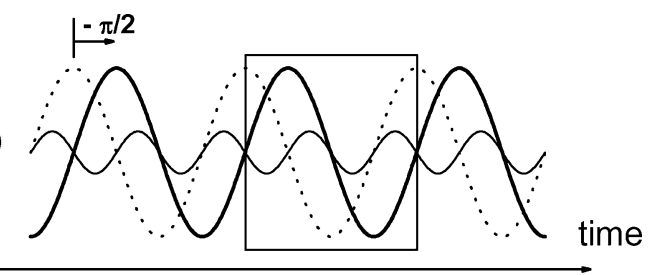

b)

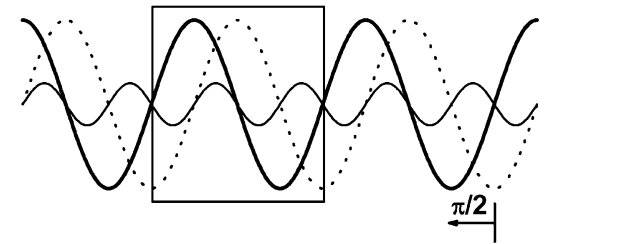

Fig. 11. Schematic representation of the voltage waveforms evolution at varactors leads when the phase of the injected signal changes in $\pi$. The dashed line corresponds to the fundamental voltage waveform just at the time when the input phase changes. This waveform can evolve in two ways to again reach the steady-state conditions: (a) by decreasing the waveform phase in $\pi / 2$ or (b) by increasing the waveform phase in the same amount. a)

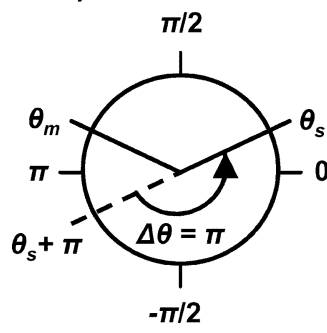

b)

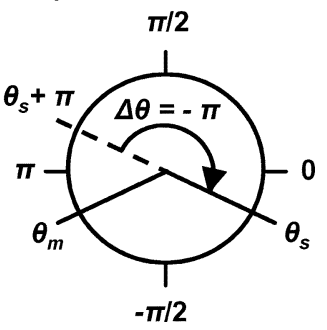

Fig. 12. Schematic representation of the evolution of angle $\theta$ when the input phase changes in $\pi$. Label (a) identifies the case when the steady-state value $\theta_{s}>0$ and (b) the case $\theta_{s}<0$.

Now let us assume that the phase of the injected signal $\psi$ changes in $\pi$. The locking phase conditions are no longer satisfied, thus, the output phase of the ILO changes to again reach the steady state. As is shown in Fig. 11, the change in the output phase $\Phi$ could be either $-\pi / 2$ or $\pi / 2$ indistinctly.

According to our previous analysis of the ILO's dynamics, when the phase $\psi$ of the input signal changes in $\pi$, the angle $\theta$ changes from it steady-state value $\theta_{s}$ to $\theta_{s}+\pi$. Depending on whether the value of $\theta_{s}$ is positive (i.e., $f_{r}>f_{\text {lock }}$ ) or negative (i.e., $f_{r}<f_{\text {lock }}$ ), the transient of $\theta(t)$ back to the steady-state conditions will consist in an increase $\Delta \theta=\pi$ or a decrease $\Delta \theta=-\pi$, respectively. This situation is shown in Fig. 12 . Taking into account (5), an increment $\Delta \theta$ leads to an increment of the output phase of the oscillator $\Delta \Phi=\Delta \theta / 2$. Hence, the response of a phase change of the BPSK input signal is a change of the phase of the oscillator output $\Delta \Phi=\pi / 2$ when $f_{r}>f_{\text {lock }}$ or $\Delta \Phi=-\pi / 2$ when $f_{r}<f_{\text {lock }}$.

Let us now consider the circuit shown in Fig. 13. A BPSK signal of frequency $2 \cdot f_{\text {lock }}$ is injected to both ILOs, e.g., using a power splitter. The total injected power is assumed to be enough to assure frequency and phase locking of both oscillators. The varactors of $\mathrm{ILO}_{1}$ are biased so that the free-running frequency $f_{r 1}>f_{\text {lock }}$. The varactors of $\mathrm{ILO}_{2}$ are also biased so that $f_{r 2}<$ $f_{\text {lock }}$. Finally, the outputs of both $\mathrm{ILO}_{1}$ and $\mathrm{ILO}_{2}$ are combined together using a power combiner. 


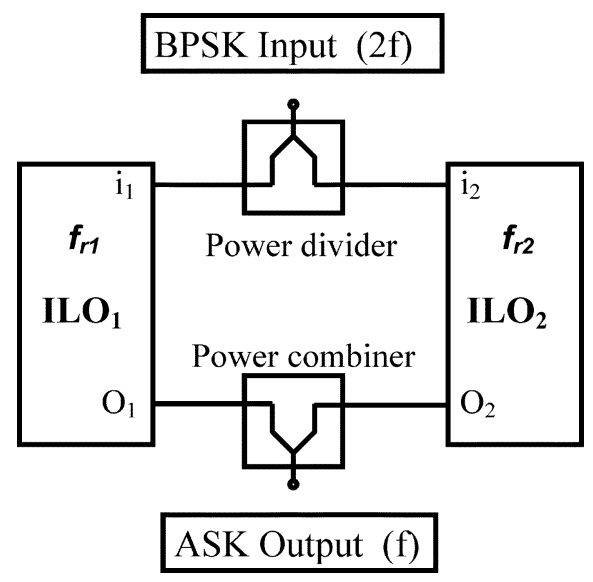

Fig. 13. Circuit block diagram of the proposed BPSK to ASK converter.

As starting point for our analysis of the BPSK to ASK conversion, and without loss of generality, we can assume that the output phase of the first oscillator $\Phi_{1}$ is the solution of (5) with $\theta(t)=\theta_{s 1}$ (i.e., the steady-state value of $\theta(t)$ for the first oscillator) and $\psi=\pi$, i.e.,

$$
\Phi_{1}=\frac{\theta_{s 1}}{2}+\frac{\pi}{4}, \quad 0<\theta_{s 1}<\frac{\pi}{2} .
$$

Analogously,

$$
\Phi_{2}=\frac{\theta_{s 2}}{2}+\frac{\pi}{4}, \quad-\frac{\pi}{2}<\theta_{s 2}<0
$$

where $\Phi_{2}$ is the output phase and $\theta_{s 2}$ is the steady-state value of $\theta(t)$ for $\mathrm{ILO}_{2}$. Under these conditions, the outputs of both oscillators $O_{1}$ and $O_{2}$ can be expressed as

$$
\begin{aligned}
& O_{1}(t)=A_{1} \exp \left\{j\left(2 \pi f_{\text {lock }} t+\frac{\theta_{s 1}}{2}+\frac{\pi}{4}\right)\right\} \\
& O_{2}(t)=A_{1} \exp \left\{j\left(2 \pi f_{\text {lock }} t+\frac{\theta_{s 2}}{2}+\frac{\pi}{4}\right)\right\} .
\end{aligned}
$$

This situation is described in the phasor diagram of Fig. 14(a). At the output of the power combiner, they will mainly constructively interfere leading to maximum output amplitude

$$
|\Sigma|=A_{1} \sqrt{2} \sqrt{1+\cos \left(\frac{\left(\theta_{s 1}-\theta_{s 2}\right)}{2}\right)} .
$$

If the phase of the BPSK input signal $\psi$ changes in $\pi$, the output phase of $\mathrm{LO}_{1}$ changes in $\pi / 2\left(f_{r 2}>f_{\text {lock }}\right)$, while the output phase of $\mathrm{ILO}_{2}$ changes in $-\pi / 2\left(f_{r 2}<f_{\text {lock }}\right)$. Similarly to (27), the outputs are given by

$$
\begin{aligned}
& O_{1}(t)=A_{1} \exp \left\{j\left(2 \pi f_{\text {lock }} t+\frac{\theta_{s 1}}{2}+\frac{\pi}{4}+\frac{\pi}{2}\right)\right\} \\
& O_{2}(t)=A_{1} \exp \left\{j\left(2 \pi f_{\text {lock }} t+\frac{\theta_{s 2}}{2}+\frac{\pi}{4}-\frac{\pi}{2}\right)\right\} .
\end{aligned}
$$

However, both outputs will now mainly destructively interfere, leading to minimum amplitude at the output of the power combiner

$$
|\Sigma|=A_{1} \sqrt{2} \sqrt{1-\cos \left(\frac{\left(\theta_{s 1}-\theta_{s 2}\right)}{2}\right)}
$$

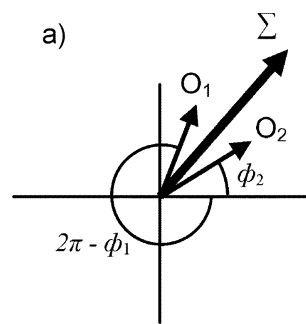

c)

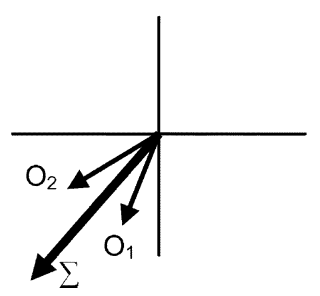

b)

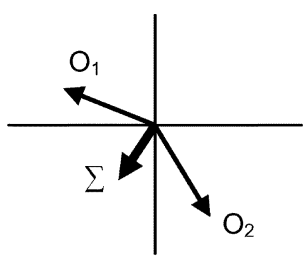

d)

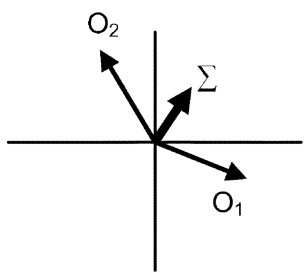

Fig. 14. Phasor diagram of the BPSK to ASK converter circuit. Label (a) corresponds to an arbitrary initial condition for which the superposition of the outputs $O_{1}$ and $O_{2}$ give a maximum and (b)-(d) correspond to successive changes in $\pi$ of the input phase.

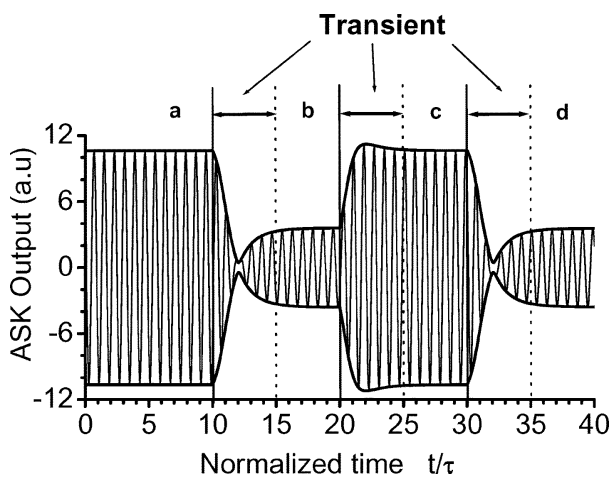

Fig. 15. Output waveform of the converter circuit of Fig. 13 in response to successive changes in $\pi$ of the input phase. Labels (a)-(d) correspond to the equivalent phasor states in Fig. 14.

as shown in Fig. 14(b). Further changes in $\pi$ of the phase of the BPSK input signal will cause consecutive switches between minimum and maximum output amplitudes following the sequence given in Fig. 14(c), (d), (a), and (b) and so on. Hence, the resulting interference pattern reproduces the phase changes of the injected BPSK signal. In others words, the circuit diagram of Fig. 13 effectively down converts the BPSK input signal at frequency $2 \cdot f_{\text {lock }}$ into an ASK output signal at frequency $f_{\text {lock}}$.

It is noteworthy that frequencies $f_{r 1}$ and $f_{r 2}$ delimit a conversion channel. Only locking frequencies inside the range $f_{r 2}<$ $f_{\text {lock }}<f_{r 1}$ correspond to BPSK input signals, which are properly converted into ASK signals. Locking frequencies outside this range will cause phase changes at the output of both oscillators of $-\pi / 2\left(f_{r 1}<f_{\text {lock }}\right.$ and $\left.f_{r 2}<f_{\text {lock }}\right)$ or $\pi / 2\left(f_{r 1}>f_{\text {lock }}\right.$ and $\left.f_{r 2}>f_{\text {lock }}\right)$ when the input phase changes in $\pi$. Consequently, no interference pattern will be generated.

\section{B. Bit Rate Estimation}

Fig. 15 shows an example of the output waveform of the converter circuit of Fig. 13 as a function of the normalized time $t / \tau$. The time-domain output signals of both oscillators have been 


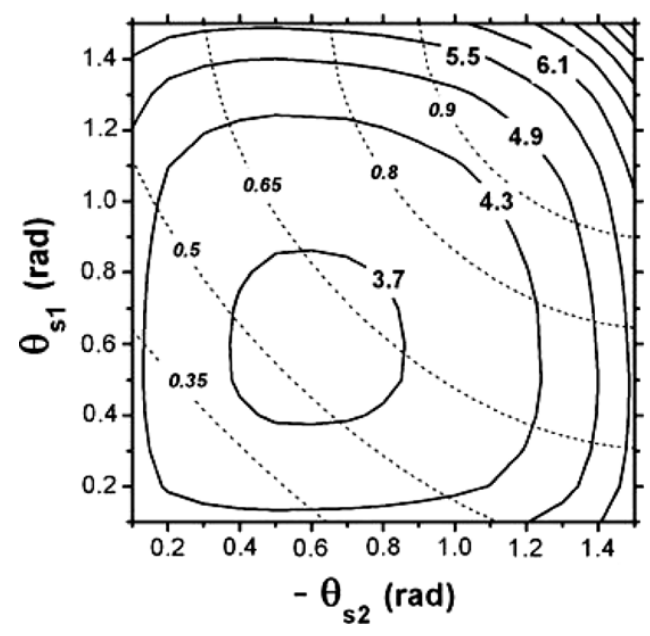

Fig. 16. Continuous closed lines are the contours of constant normalized transient time. Dashed lines are the contours of constant normalized conversion channel. Both set of curves are represented in the $\left(\theta_{s 1},-\theta_{s 2}\right)$ plane.

computed using an arbitrary carrier frequency $f_{\text {lock }}$ and the analytical expressions of the phase behavior (14) to (24). This figure shows the resulting interference pattern in response to consecutive phase changes of the BPSK input signal. Here, input phase changes take place every ten normalized time units. After each input phase change, a transient response of the output can be observed lasting approximately five normalized time units. For a better understanding, letter labels in this figure correspond to the different steady states of Fig. 14.

It is noteworthy that the conversion dynamics of the new converter circuit is significantly different than that of the classical demodulators based on synchronization loops. In the new approach, the BPSK to ASK converter does not lock to recover the carrier and then demodulates, but relocks for every single bit (i.e., phase change of the input signal). Consequently, the new demodulation scheme will be very useful in burst mode communication where the classical approach fails due to the usually large locking time.

The transient response of the converter circuit will be one of the limiting factors of the maximum achievable bit rate. Only BPSK signals having a bit rate $M<1 / t_{r}$ can be properly downconverted into ASK signals, with $t_{r}$ being the total transient time. Using normalized time units, the transient time $t_{r}$ only depends on the steady-state values $\theta_{s 1}$ and $\theta_{s 2}$. Fig. 16 shows the projection of constant $t_{r} / \tau$ contours (continuous lines) on the plane $\left(\theta_{s 1},-\theta_{s 2}\right)$. Transient times have been evaluated as the required time to achieve $95 \%$ of the total output phase change after a sudden input phase change of $\pi$.

In Fig. 16, there are dashed curves corresponding to constant values of $\left(\sin \theta_{s 1}-\sin \theta_{s 2}\right) / 2$. Taking into account that $f_{r 1} \approx$ $f_{\text {lock }} \approx f_{r 2}$, it can be seen that these curves also correspond to constant values of the conversion channel width $f_{r 1}-f_{r 2}$. In fact, according to (9), one can obtain

$$
f_{r 1}-f_{r 2} \approx f_{\text {lock }} \frac{\alpha A_{2}}{4 C_{o}}\left(\sin \theta_{s 1}-\sin \theta_{s 2}\right) .
$$

For a given injected power, the maximum value of $f_{r 1}-f_{r 2}$ is achieved for $\theta_{s 1}=-\theta_{s 2}=\pi / 2$. Substituting this condition

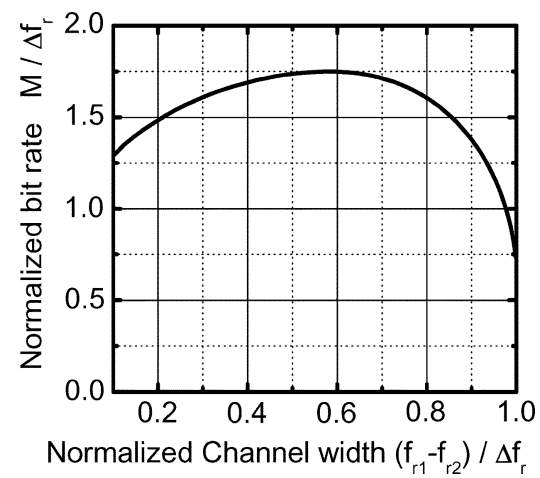

Fig. 17. Normalized bit rate as a function of the normalized channel width.

in (31), the following expression is obtained for the maximum conversion channel width:

$$
\Delta f_{r}=\left|f_{r 1}-f_{r 2}\right|_{\max } \approx f_{\text {lock }} \frac{\alpha A_{2}}{2 C_{o}} \approx \frac{1}{2 \pi \tau} .
$$

By combining (31) and (32), one finally obtains the following expression for the normalized channel width:

$$
\frac{f_{r 1}-f_{r 2}}{\Delta f_{r}} \approx \frac{\left(\sin \theta_{s 1}\right)-\sin \theta_{s 2}}{2} .
$$

According to data in Fig. 16, for a given conversion channel width, the minimum transient response is observed when $\theta_{s 1}=$ $-\theta_{s 2}$. Under such condition, the locking frequency $f_{\text {lock }}$ is centered between $f_{r 1}$ and $f_{r 2}$. Moreover, the absolute minimum of the transient time is found to be approximately 3.6 normalized time units for $\theta_{s 1}=-\theta_{s 2} \approx 0.6$.

Fig. 17 shows the normalized bit rate as a function of the normalized channel width. It is noteworthy that a maximum bit rate of approximately $1.75 \Delta f_{r}$ is achieved for a conversion channel width equal to the $55 \%-60 \%$ of the maximum value $\Delta f_{r}$. Equivalently, for a given conversion channel width $f_{r 1}-f_{r 2}$, the maximum achievable bit rate will be approximately $3 \cdot\left(f_{r 1}-f_{r 2}\right)$ provided the converter is operating under optimal conditions.

\section{Conversion Dynamics}

The above discussion of the conversion dynamics has been done considering a steep phase change of the BPSK input signal. However, this phase change usually takes place during a certain transition time $t_{0}$. Among the multiple possible trajectories going from one symbol to the other of a BPSK signal, we have consider in detail three simple cases, which are shown in Fig. 18. The first one, labeled $a$, corresponds to a constant decrease of the injected amplitude, a phase change of $\pi$ when the amplitude reaches zero, and a constant amplitude increase up to the initial value. This trajectory is the result of the multiplication of a carrier signal with a quasi-step function from -1 to 1 or vice versa having a certain rise or fall time, respectively. The second trajectory, labeled $b$, corresponds to a continuous phase increase in $\pi$ during the transition time, keeping constant the signal amplitude. Finally, the third trajectory corresponds to a constant signal amplitude and a continuous phase decrease in $-\pi$ during the same time.

We next analyze these three cases through the evaluation of $\mathrm{ILO}_{1}$ in Fig. 13, i.e., the one with the condition $f_{r 1}>f_{\text {lock }}$. 


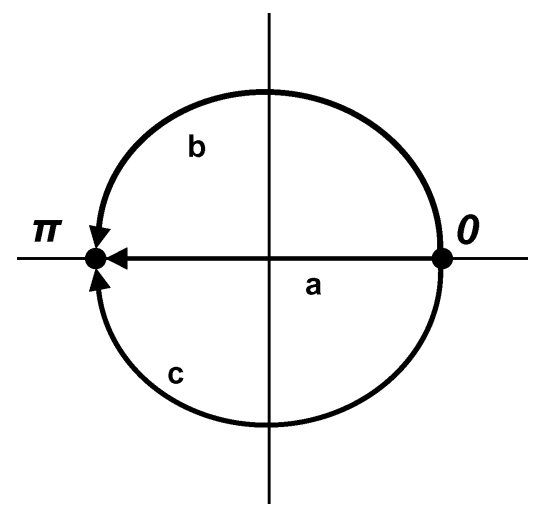

Fig. 18. Simple trajectories in the constellation diagram between both symbols of a BPSK signal.

1) Case a: The study of the dynamic behavior of the converter in response to a phase change of the BPSK signals must be carried out considering three different stages. First, the amplitude of the injected signal decreases from its initial value $A_{2}$ to zero during a normalized time $t_{0}$. Second, the phase of the injected signal $\psi$ suddenly changes in $\pi$ and the amplitude increases from zero to the initial value $A_{2}$, also during a time $t_{0}$. Finally, the converter system evolves until an equilibrium condition is reached once again.

Assuming a linear decrease of the injected amplitude, the dynamics of the first stage is governed by the following differential equation:

$$
\frac{d \theta_{I}}{d t}=4 \pi\left(f_{r 1}-f_{\text {lock }}\right)+-\pi f_{r 1} \frac{\alpha A_{2}}{C_{o}}\left(1-\frac{t}{t_{o}}\right) \sin \left(\theta_{I}(t)\right)
$$

where $t_{o}$ is the fall time. The domain of integration is $\left(0, t_{o}\right)$ and the initial condition is $\theta_{I}(0)=\theta_{s 1}$.

For the second stage, the differential equation to be solved is given by

$\frac{d \theta_{I I}}{d t}=4 \pi\left(f_{r 1}-f_{\mathrm{lock}}\right)+-\pi f_{r 1} \frac{\alpha A_{2}}{C_{o}}\left(\frac{t}{t_{o}}-1\right) \sin \left(\theta_{I I}(t)\right)$

being the integration domain $\left(t_{o}, 2 t_{o}\right)$ and the initial conditions $\theta_{I I}\left(t_{0}\right)=\theta_{I}\left(t_{0}\right)+\pi$.

Finally, the differential equation for the third stage is given by (8), which can be rewritten as

$$
\frac{d \theta_{I I I}}{d t}=4 \pi\left(f_{r 1}-f_{\text {lock }}\right)-\pi f_{r 1} \frac{\alpha A_{2}}{C_{o}} \sin \left(\theta_{I I I}(t)\right)
$$

with an integration domain $\left(2 t_{o}, \infty\right)$ and the initial condition $\theta_{I I I}\left(2 \cdot t_{0}\right)=\theta_{I I I}\left(2 \cdot t_{0}\right)$.

Once the solutions for the three stages are known, the output phase change $\Delta \Phi(t)$ of the converter can be evaluated. According to (5), one obtains

$$
\begin{aligned}
\Delta \Phi(t)=\frac{\theta_{I}(t)-\theta_{s 1}}{2}, & 0 \leq t \leq t_{o} \\
\Delta \Phi(t)=\frac{\theta_{I I}(t)-\theta_{s 1}-\pi}{2}, & t_{o} \leq t \leq 2 t_{o} \\
\Delta \Phi(t)=\frac{\theta_{I I I}(t)-\theta_{s 1}-\pi}{2}, & t \geq 2 t_{o}
\end{aligned} \mid .
$$

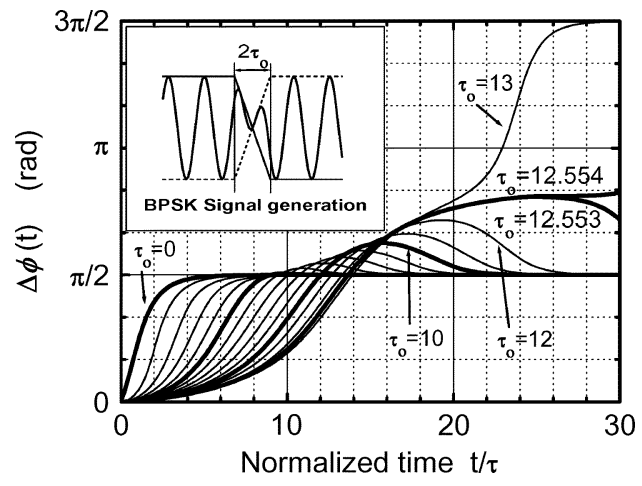

Fig. 19. Plot of output phase $\Delta \Phi(t)$ as a function of the normalized time for different values of the transit time $\tau_{0}$. The insert shows the BPSK waveform corresponding to the trajectory labeled " $a$ " in Fig. 18.

Equations (34) and (35) have been solved numerically, whereas the solution of (36) is given by (16)-(18). The same procedure can be applied for solving the output phase of $\mathrm{ILO}_{2}$, the one with $f_{r 2}<f_{\text {lock}}$. From the symmetry of the problem, similar expressions will be found.

Fig. 19 shows the global solution of $\Delta \Phi(t)$ in the case $\theta_{s 1}=0.6$ and for several values of the normalized transit time $\tau_{0}=t_{0} / \tau$. The inset in this figure illustrates the generation procedure of the input BPSK signal. It should be noted that the output phase change $\Delta \Phi(t)$ shows a bifurcation behavior depending on the normalized transit time $\tau_{0}$. If $t_{0}$ is smaller than the bifurcation time $t_{b}$ (in the example shown in Fig. 19, $\left.t_{b} / \tau=12.5535 \ldots\right)$, then the output phase shift is $\pi / 2$, as expected when $f_{r 1}>f_{\text {lock }}$ (i.e., $\theta_{s 1}>0$ ). On the contrary, if the transit time is injected, amplitude increases above the locking threshold. On the contrary, for a long time interval of the oscillators in the free-running state, a wrong final output phase will be reached.

Further increase of the transit time reveals the existence of additional bifurcation times, which delimits zones of right and wrong behavior of the converter. Accordingly, the first bifurcation time must be understood as the maximum acceptable fall and/or rise time of the trapezoidal waveform used to generate the BPSK signal. Bifurcation phenomena in harmonic-injected dividers have been analyzed by other authors [15]. However, the analysis has been carried out taking into account only the injected power and not the input phase change dynamics.

2) Cases b and c: In these cases, we have to take into account two different stages to analyze the dynamics of the converter in response to a phase change of the BPSK signals. First, the phase of the injected signal increases or decreases linearly in $\pi$ during a certain transit time $2 \cdot t_{o}$ (factor 2 is included for comparative purposes with the previous case). Second, the converter system evolves until an equilibrium condition is again reached.

According to (5)-(7), the dynamics of the first stage is governed by the differential equations

$$
\frac{d \theta_{I}}{d t}=4 \pi\left(f_{r 1}-f_{\text {lock }}\right)-\frac{d \psi}{d t}-\pi f_{r 1} \frac{\alpha A_{2}}{C_{o}} \sin \left(\theta_{I}(t)\right)
$$

that can be rewritten as

$$
\frac{d \theta_{I}}{d t}=4 \pi\left(f_{r 1}-f_{\mathrm{eq}}\right)-\pi f_{r 1} \frac{\alpha A_{2}}{C_{o}} \sin \left(\theta_{I}(t)\right)
$$




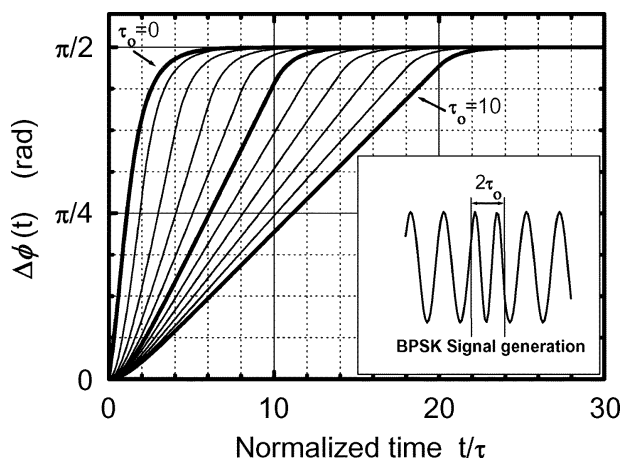

Fig. 20. Plot of output phase $\phi(t)$ as a function of the normalized time for different values of the transit time $\tau_{0}$. The insert shows the BPSK waveform corresponding to the trajectory labeled " $b$ " in Fig. 18.

with being $f_{\text {eq }}$ an equivalent injection frequency, which is given by

$$
f_{\text {eq }}=f_{\text {lock }}+\frac{1}{4 \pi} \frac{d \psi}{d t}=f_{\text {lock }}+\frac{1}{4 \pi} \frac{\pi}{2 t_{0}} .
$$

The integration domain of (39) is $\left(0,2 t_{o}\right)$, and the initial condition is $\theta_{I}(0)=\theta_{s 1}$ and $\psi(0)=0$ (this last value can be arbitrarily assigned). Depending on the value of $f_{\text {eq }}$, the solution will be one of those described in Section II-C.

According to the previous discussion, the transit between symbols of the BPSK signal has to be interpreted just as a change in the injection frequency from $f_{\text {lock }}$ to $f_{\text {eq }}$.

For the second stage, the equation to solve is given by (8), which, in this case, can be expressed as

$$
\frac{d \theta_{I I}}{d t}=4 \pi\left(f_{r 1}-f_{\mathrm{eq}}\right)-\pi f_{r 1} \frac{\alpha A_{2}}{C_{o}} \sin \left(\theta_{I I}(t)\right)
$$

with the integration domain being $\left(2 \cdot t_{o}, \infty\right)$ and the initial condition being $\theta_{I I}\left(2 \cdot t_{0}\right)=\theta_{I I}\left(2 \cdot t_{0}\right)$.

Finally, the converter's output phase change $\Delta \Phi(t)$ is given by

$$
\begin{array}{cc}
\Delta \Phi(t)=\frac{\theta_{I}(t)-\theta_{s 1}+\psi(t)}{2}, & 0 \leq t \leq 2 t_{o} \\
\Delta \Phi(t)=\frac{\theta_{I I}(t)-\theta_{s 1} \pm \pi}{2}, & t \geq 2 t_{o}
\end{array} \mid .
$$

The plus sign applies when the phase on the input signal increases from 0 to $\pi$, while the minus sign applies when the phase decreases from 0 to $-\pi$.

Figs. 20 and 21 depict $\Delta \Phi(t)$ for $\theta_{s 1}=0.6$ and several values of the normalized transit time $\tau_{0}=t_{0} / \tau$, assuming a linear variation of $\psi(t)$ from 0 to $\pm \pi$, respectively. In both cases, the inset illustrates the generation procedure of the input BPSK signal. In the first case, shown in Fig. 20, the locking frequency increases by an amount equal to $t_{o} / 8$ during the transit time, whereas in the second case in Fig. 21, the locking frequency decreases by the same amount. It is important to note that the bifurcation behavior is only observed in the second case.

To understand these results, we have to take into account that trajectories $b$ and $c$ are equivalent when the transit time is very short. In that case, the injected oscillators only react to the final value of phase $\psi$, which is the same in both cases. This can be observed in both Figs. 20 and 21 as an output phase change in

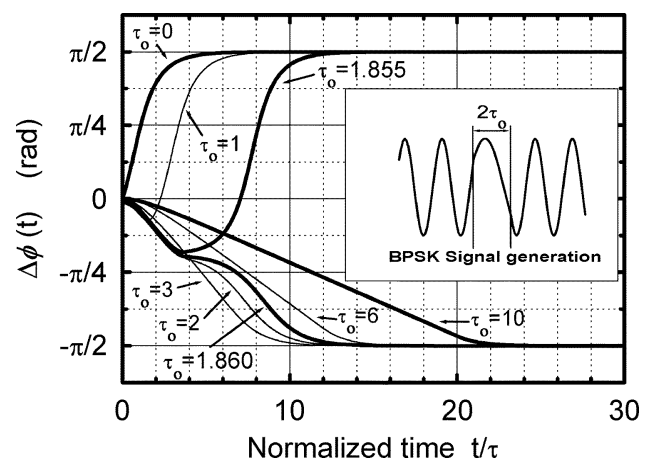

Fig. 21. Plot of output phase as a function of the normalized time for different values of the transit time $\tau_{0}$. The insert shows the BPSK waveform corresponding to the trajectory labeled " $c$ " in Fig. 18.

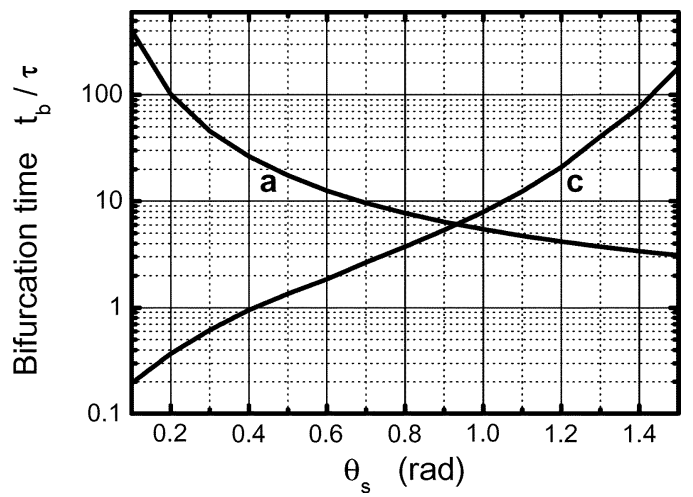

Fig. 22. Normalized bifurcation time as a function of angle $\theta_{s}$ for trajectories labeled " $a$ " and " $c$ " in Fig. 18.

$\pi / 2$, as expected when $f_{r}>f_{\text {lock }}$ (i.e., $\theta_{s 1}>0$ ). On the contrary, if the transit time is long enough, the injected oscillators follow the input phase changes and then the final value of the output phase will depend on the input phase trajectory. In case $b, \Delta \Phi=\pi / 2$ and no bifurcation appears. However, in case $c$, $\Delta \Phi=\pi / 2$ and the bifurcation behavior is observed. Opposite results would be obtained for $\mathrm{ILO}_{2}$ because $f_{r}<f_{\text {lock }}$ (i.e., $\theta_{s 2}>0$ ) is considered. In any case, the bifurcation time must be considered the maximum acceptable transit time between symbols of the BPSK input signal.

To conclude, Fig. 22 shows the normalized bifurcation time as a function of angle $\theta_{s}$ for trajectories $a$ and $c$. Note the important differences in behavior related to the different nature of the bifurcation phenomena. BPSK signals generated using a schema in accordance with trajectory $a$ will be better demodulated using strong injected oscillators (i.e., small values of $\theta_{s}$ ). On the contrary, BPSK signals generated according to trajectories $b$ or $c$ will be better demodulated using weak injected oscillators.

\section{CONCLUSION}

A new method and circuit to convert BPSK signals into ASK signals based on the use of second harmonic ILOs has been presented.

First, the second harmonic ILOs have been analyzed in detail. Their dynamics in response to phase changes of the injected signal has been studied exhaustively. Hence, an analytical expression, describing the oscillator response to phase changes of 
the BPSK input signal, has been obtained for all possible locking conditions.

Second, the conversion mechanism, based on frequency and phase synchronization and interference phenomena, has been studied in detail. As a result, the existence of a conversion channel, controlled by external bias, has been pointed out. Moreover, the out-of-channel rejection of the converter has been discussed in detail.

In addition, the dynamics of the conversion mechanism has also been extensively analyzed. The maximum achievable modulation bit rate has been studied as a function of the locking conditions of the converter. From this study, the optimum operating conditions of the converter have been derived, and a relationship between the conversion channel width and the maximum bit rate has been established.

Finally, the limitations of the conversion process related to the characteristics of the BPSK signal (i.e., nonnull transit time from one symbol to another) have been considered. Three relevant cases have been studied exhaustively and, as a result, the existence of a bifurcation behavior of the converter response has been evidenced. From this, maximum acceptable transit times between symbols of the input BPSK signal have been obtained.

\section{REFERENCES}

[1] B. Sklar, Digital Communication Fundamentals and Applications. Upper Saddle River, NJ: Prentice-Hall, 1988.

[2] J. P. Costas, "Synchronous communications," Proc. IRE, vol. 47, pp. 2058-2068, 1959.

[3] G. L. Do and K. Feher, "An ultra-fast carrier recovery versus traditional synchronizers," IEEE Trans. Broadcast., vol. 42, no. 1, pp. 42-49, Mar. 1996.

[4] S. Mirabbasi, S. Gazor, and K. Martin, "A wide-band carrier-recovery system for multilevel QAM signals," in Proc. IEEE Int. Circuits Systems Symp., 2000, pp. IV-661-IV-664.

[5] H. Sari and S. Moridi, "New phase and frequency detectors for carrier recovery in PSK and QAM systems," IEEE Trans. Commun., vol. 36, no. 9, pp. 1035-1043, Sep. 1988.

[6] J. M. Lopez-Villegas, J. G. Macias, J. Cabanillas, J. J. Sieiro, J. A. Osorio, J. Samitier, J. Bausells, J. Montserrat, and E. Cabruja, "BPSK to ASK converter for RF digital communications," in IEEE Radio Frequency Integrated Circuits Symp. Dig., 2003, pp. 643-646.

[7] B. Van der Pol, "Forced oscillations in a circuit with nonlinear resistance," Phil. Mag., vol. 3, pp. 65-80, Jan. 1927.

[8] R. Adler, "A study of locking phenomena in oscillators," Proc. IRE, vol. 34, no. 6, pp. 351-357, Jun. 1946.

[9] - "A study of locking phenomena in oscillators," Proc. IEEE, vol. 61, no. 10, pp. 1380-1385, Oct. 1973.

[10] M. T. Jezewski, "An approach to the analysis of injection-locked oscillators," IEEE Trans. Circuits Syst., vol. CS-21, no. 5, pp. 395-401, May 1974.
[11] E. F. Calandra and A. M. Sommariva, "Stability analysis of injectionlocked oscillators in their fundamental mode of operation," IEEE Trans. Microw. Theory Tech., vol. MTT-29, no. 11, pp. 1137-1143, Nov. 1981.

[12] H. R. Rateg and T. H. Lee, "Superharmonic injection-locked frequency dividers," IEEE J. Solid-State Circuits, vol. 34, no. 6, pp. 813-821, Jun. 1999.

[13] S. Verma, H. R. Rateg, and T. H. Lee, "A unified model for injectionlocked frequency dividers," IEEE J. Solid-State Circuits, vol. 38, no. 6, pp. 1015-1027, Jun. 2003.

[14] J. M. Lopez-Villegas, J. G. Macias, J. A. Osorio, J. Cabanillas, J. J. Sieiro, J. Samitier, and N. Vidal, "Continuous phase shift of sinusoidal signals using injection locked oscillators," IEEE Microw. Wireless Compon. Lett., vol. 15, no. 5, pp. 312-314, May 2005.

[15] F. Ramirez, M. E. de Cos, and A. Suárez, "Nonlinear analysis tools for the optimized design of harmonic-injection dividers," IEEE Trans. Microw. Theory Tech., vol. 51, no. 6, pp. 1752-1762, Jun. 2003.

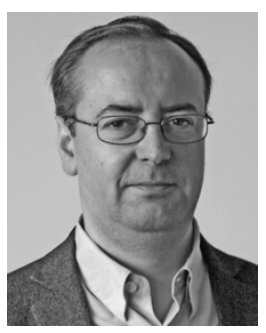

José María López-Villegas (M'93-SM'03) was born in Barcelona, Spain, in 1962. He received the Physics degree and Ph.D. degree in physics from the University of Barcelona, Barcelona, Spain, in 1985 and 1990, respectively.

From 1985 to 1987, he was Visiting Research Fellow with the Laboratoire d'Electronic Philips (LEP), Paris, France, where he was involved in the electrical characterization of III-V compound semiconductor devices. In 1990, he joined the Electronic Department, University of Barcelona, initially as an Assistance Professor and then as a permanent Professor Titular. He is currently the Head of the RF Group, Electronics Department, University of Barcelona. His research and development activities are focused on the design optimization and test of RF systems and circuits performed using silicon technologies. Within this field, he is particularly interested in the modeling and optimization of integrated inductors and transformers for RFICs applications and in the development of new homodyne transceiver architectures.

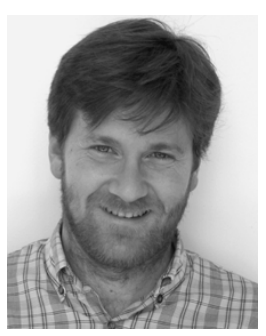

Javier Jose Sieiro Cordoba (M'04) was born in Barcelona, Spain, in 1971. He received the Physics degree, M.S. degree in electronic engineering, and Ph.D. degree in physics from the University of Barcelona, Barcelona, Spain, in 1995, 1999, and 2001, respectively.

From 2002 to 2003, he was a member of the Electronic Components Technology and Materials (ECTM) Group, Delft University of Technology, Delft, The Netherlands, where he was involved with the modeling of passive components and the design of RFIC circuits. Since 2003, he has been with the Department of Electronics, University of Barcelona, where here is currently an Associate Professor. His research interest are the modeling of passive components and the design of homodyne transceiver architectures. 